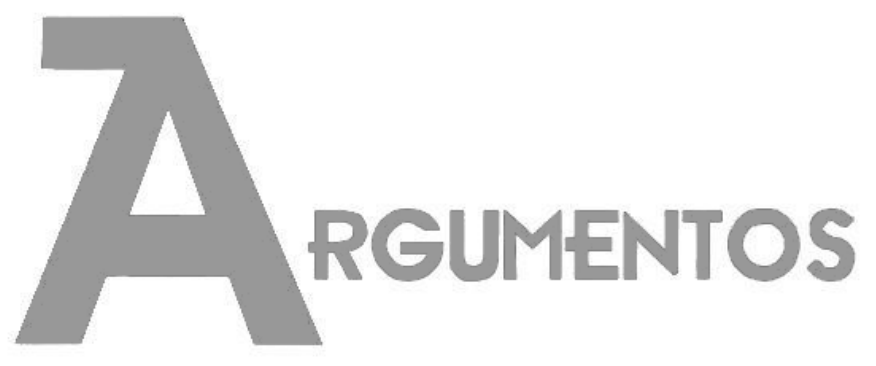

Vol. 18, n. 2, jul./dez. 2021 ISSN: 2527-2551 (online)

\title{
La modernización en el fútbol uruguayo: tensiones surgidas en torno al deterioro de los clubes y al profesionalismo en la segunda mitad del siglo XX
}

Gastón Laborido ${ }^{1}$

Recebido em: 21/03/2021

Aprovado em: 22/05/2021

\begin{abstract}
Resumen: El fenómeno de la modernización del y en el fútbol es un proceso relativamente nuevo que ha generado debates a partir de las transformaciones provocadas en diferentes ámbitos. Esos debates versan en torno al nuevo modelo europeizado del fútbol, al nuevo concepto de espectáculo y a una mercantilización agresiva; que tienen lógicas funcionales propias de la globalización. El fútbol uruguayo vivió en la primera mitad del siglo XX un período de bonanza y el mundo conoció a Uruguay por el fútbol. ¿Cómo actuó ese proceso modernizador en el fútbol uruguayo? ¿Cuál fue el impacto en nuestro país a partir de la década del sesenta? ¿Qué tensiones subyacen al proceso? Para explicar e interpretar el asunto se incorporan herramientas analíticas como la noción de "arena pública", que ayuda a realizar una lectura de la sociedad uruguaya a partir del mundo simbólico del fútbol.
\end{abstract}

Palabras clave: Historiografía; Fútbol; Modernización; Clubes; Deterioro.

\section{A modernização no futebol uruguaio: tensões surgidas em torno à deterioração dos clubes e do profissionalismo na segunda metade do século XX}

Resumo: O fenômeno da modernização do/no futebol é um processo relativamente novo que tem gerado debates a partir das transformações causadas em diferentes áreas. Esses debates giram em torno do novo modelo europeizado de futebol, o novo conceito de entretenimento e uma mercantilização agressiva; que têm lógicas funcionais típicas da globalização. Na primeira metade do século XX, o futebol uruguaio viveu um período de boom e o mundo conheceu o Uruguai através desse esporte. Como funcionou esse processo de modernização do futebol uruguaio? Qual foi o impacto em nosso país a partir dos anos sessenta? Quais tensões estão por

\footnotetext{
${ }^{1}$ Profesor de Historia de enseñanza media - Uruguay (DGES). Profesor de Historia del Deporte, Ed. Física y Recreación (IUACJ). Integrante del Grupo de Estudios de Fútbol del Uruguay (GREFU-UDELAR). Maestrando en Ciencias Humanas, opción Historia Rioplatense, en Facultad de Humanidades y Ciencias de la Educación (FHCE-UDELAR), Uruguay. E-mail: gaston_laborido1@hotmail.com. ORCID: https://orcid.org/0000-0003-0756-6739.
} 
Dossiê | La modernización en el fútbol uruguayo: tensiones surgidas en torno al deterioro de los clubes y al profesionalismo en la segunda mitad del siglo XX (LABORIDO, Gastón)

trás do processo? Para explicar e interpretar o assunto, são incorporadas ferramentas analíticas, como a noção de "arena pública", que ajudam a fazer uma leitura da sociedade uruguaia a partir do mundo simbólico do futebol.

Palavras-chave: Historiografia; Futebol; Modernização; Clubes; Deterioração.

\section{The modernization of uruguayan soccer, stress brought about due to clubs deterioration and to professionalism on the second half of the twentieth century}

Abstract: The phenomenon of modernization of and in footballis a relatively new process that has generated debates based on the transformations caused in different areas. These debates are about the new Europeanized football model, to the new concept of entertainment and an aggressive commodification; that have functional logics typical of globalization. Uruguay an football experienced a boom period in the first half of the 20th century and the world got to know Uruguay through football. How did this modernizing process work in Uruguay an football? What was the impact on our country from the sixties? What tensions underlie the process? To explain and interpret the matter, analytical tolos such as the notion of "public arena" are incorporated, which helps to make a reading of Uruguay an society from the symbolic world of football.

Keywords: Historiography; Football; Modernization; Clubs; Deterioration.

\section{Introducción}

Aunque parezca una paradoja, escribir historia del deporte con una perspectiva académica y crítica sobre el fútbol uruguayo no es una tarea sencilla. Más difícil aun, es hacerlo sobre historia reciente. Cuando recibí la invitación para escribir en este dossier, dedicado al fenómeno de la modernización del y en el fútbol en América Latina acepté con gusto, aunque reconociendo que ello implicaría un gran desafío.

La pregunta que se realizarán quienes lean este texto es, ¿cómo es posible que un país con una rica historia futbolística en el siglo XX no cuente con una producción historiográfica acorde a la relevancia del tema? En diferentes encuentros regionales con estudiosos del fútbol, sobre todo con colegas argentinos y brasileños, están presentes en los intercambios estas inquietudes y búsquedas de respuestas de esta paradoja y de los vacíos historiográficos.

Es cierto que el fútbol uruguayo fue pionero en conquistas internacionales: a nivel de selección dos medallas de oro en Juegos Olímpicos, dos Mundiales de Fútbol, mientras que a nivel clubista consiguió ocho copas Libertadores entre Nacional y Peñarol. No hay dudas que por lo menos hasta la década del sesenta del siglo XX fue un modelo exitoso. Pero hubo que esperar prácticamente cien años para que las ciencias sociales produzcan discursos explicativos e interpretativos sobre fútbol. Mientras tanto, 
Dossiê | La modernización en el fútbol uruguayo: tensiones surgidas en torno al deterioro de los clubes y al profesionalismo en la segunda mitad del siglo XX (LABORIDO, Gastón)

las narrativas provenían de ámbitos fuera de los circuitos académicos, por lo general desde el periodismo y se caracterizan por tener un enfoque semejante a la ficción sentimental memorística antes que histórico.

Los cambios comenzaron a inicios del siglo XXI y desde ahí se viene investigando sobre deporte en nuestro país, aunque con una perspectiva sociológica, antropológica y en menor medida histórica. Estas nuevas perspectivas y enfoques han generado que en algunos artículos y libros específicos, se tome al fútbol como objeto de estudio.

Con la intención de analizar el tema propuesto en clave histórica, este artículo pretende incorporar categorías y herramientas conceptuales para explicar e interpretar el proceso de modernización del y en el fútbol uruguayo (proceso acentuado en la década del sesenta pero que aun hoy sigue generando impactos), colocándolo en un espacio de debate desde una perspectiva crítica. Esto supone afirmar, que el fútbol es una práctica social que genera manifestaciones en otros espacios sociales, además de constituirse como una práctica social en sí misma.

Este fenómeno es un proceso relativamente nuevo, por lo tanto los debates surgidos a partir de las transformaciones que ha provocado en diferentes ámbitos, también son relativamente nuevos. A grandes rasgos esos debates versan en torno al nuevo modelo europeizado del fútbol, al nuevo concepto de espectáculo futbolístico y a una mercantilización agresiva del fútbol. Esto generó que el fútbol transitara por una serie de cambios (el juego, la organización, difusión mediática, consumo, afición, entre otros) que están insertos en los procesos de mercantilización, hipermediatización y transnacionalización; con lógicas de funcionamiento propias de la globalización. Por lo tanto, aquí surgen las siguientes preguntas: ¿cómo actuó ese proceso modernizador en el fútbol uruguayo? ¿Cuál fue el impacto en nuestro país?

Se trató de un período caracterizado por el pasaje a nivel mundial del amteurismo al profesionalismo. "Así cunde la especialización, la aplicación creciente de la tecnología y la conversión del deporte en uno de los más importantes emprendimientos económicos. Como resultado se va dando el actual gigantismo, espectacularización y comercialismo en los Juegos Olímpicos y en los Campeonatos Mundiales" (GOMENSORO, 2015, p. 32). Frente a ese fenómeno Uruguay mantuvo su funcionamiento y organización, basado en el modelo originario de matriz batllista y el resultado fue la pérdida de lugares de privilegios anteriores. 
Dossiê | La modernización en el fútbol uruguayo: tensiones surgidas en torno al deterioro de los clubes y al profesionalismo en la segunda mitad del siglo XX (LABORIDO, Gastón)

El artículo que presento, tiene dos grandes objetivos. El primero, es colocar en la agenda académica un posible abordaje de la historia del fútbol uruguayo, con herramientas analíticas que nos ayuden a realizar una lectura de la sociedad uruguaya a partir del mundo simbólico del fútbol. Entendemos que la Historia del Deporte es un campo profesional de investigación histórica que a nivel regional se viene consolidando y que es necesario pensar en las posibilidades que nos brinda su estudio.

El segundo objetivo, es generar un marco explicativo de un tema-problema que se circunscribe en el contexto de la modernización del futbol. Es obvio que el proceso tiene múltiples consecuencias generando diversificación de debates producto de los cambios económicos, sociales, culturales que generan. En este nuevo escenario es que nos preguntamos, ¿Qué tensiones subyacen al proceso de modernización en el fútbol uruguayo?

A los efectos de organizar este trabajo, es que se presentan dos partes. En la primera se pretende realizar una aproximación a cuestiones teóricas y metodológicas de la Historia del Deporte como campo de conocimiento, rescatando categorías analíticas para pensar acerca del fenómeno deportivo. Así es que concebimos al fenómeno del fútbol como "arena pública", noción desarrollada por Eduardo Archetti (1985), utilizada para mostrar como el deporte era un espacio privilegiado para indagar ciertos elementos que la sociedad y la cultura ponen en escena y que pueden ser usadas como medio para reflexionar sobre la sociedad uruguaya.

La segunda parte del trabajo, pretende interpretar y explicar algunas de las tensiones que subyacen al proceso de modernización en el fútbol uruguayo: los inicios del estancamiento y el deterioro de los clubes sociales a partir de los años sesenta. Los clubes barriales han dejado de tener potencia instituyente de espacio colectivo y de lazo comunitario; dando paso a un nuevo modelo de club, con otros significados y nuevas formas de organización.

\section{Los abordajes del deporte en clave histórica en el Uruguay}

La Historia del Deporte como campo profesional de investigación histórica a nivel internacional se ha ido consolidando en las últimas décadas del siglo XX y en gran medida tiene relación con la configuración de la Nueva Historia Cultural. La historia 
Dossiê | La modernización en el fútbol uruguayo: tensiones surgidas en torno al deterioro de los clubes y al profesionalismo en la segunda mitad del siglo XX (LABORIDO, Gastón)

cultural aglutinó la actividad académica en países de ámbito anglosajón, tuvo una excelente acogida en el mundo académico norteamericano y desde allí se ha ido extendiendo a otras tradiciones historiográficas. Este fenómeno es conocido como el "cultural turn", tendencia que colocó su atención por el lenguaje y sus estructuras más profundas, alejándola de la tradición marxista y de otras ciencias sociales como la sociología y la ciencia política.

Tiene como referentes teóricos a Hayden White, Michel Foucault, Roland Barthes, Pierre Bourdieu, Jacques Derrida, Thomas Kuhn, Richard Rorty, Marshall Sahlins y Raymond Williams. Todos ellos provenientes de diferentes ciencias sociales (la antropología, la filosofía, la sociología y la lingüística) y de diferentes tendencias intelectuales (posmarxismo, postestructuralismo, deconstruccionismo $\mathrm{y}$ posmodernismo). Es en este sentido, que la nueva historia cultural tiene una enorme capacidad de aglutinación y consenso epistemológico.

La Nueva Historia Cultural se consolidó a través de un complejo proceso de criba epistemológica y haberse apropiado de las ideas de Clifford Geertz y la moderna antropología. Geertz (1926-2006) fue un antropólogo estadounidense, considerado uno de los más influyentes de su generación. Realizó sus trabajos de campo en Indonesia (Bali y Java) y Marruecos y escribió destacadas monografías y realizó estudios históricos. En consecuencia, para la Nueva Historia Cultural, la cultura es la fuente fundamental de comprensión histórica, esto supone, que el mundo puede ser leído como un texto.

Según Victor Andrade de Melo y Rafael Fortes (2010) la Historia del Deporte es "hija" de la Nueva Historia Cultural, pero afirman que se fue envolviendo y relacionando más a las premisas teóricas de la historia social. De esta manera, los investigadores de historia del deporte pasaron a prestar más atención a las potencialidades que brinda el campo, sobre todo tomando aportes provenientes desde la Antropología. Este fenómeno se dio mayoritariamente en países centrales y comenzaron a concebir al deporte como objeto de estudio.

Mientras tanto, ¿̇qué ha ocurrido con las historias del deporte uruguayo? Al igual que en América Latina, el deporte sufrió una desatención por parte de las ciencias sociales. En este sentido, 
Dossiê | La modernización en el fútbol uruguayo: tensiones surgidas en torno al deterioro de los clubes y al profesionalismo en la segunda mitad del siglo XX (LABORIDO, Gastón)

el deporte permaneció obturado hasta fechas muy recientes como una posibilidad de discurso letrado, a excepción de la narrativa ficcional (y con cierta parvedad), del costumbrismo y del periodismo especializado. En este último caso, inversamente, el desborde productivo apunta hacia la saturación (ALABARCES, 2000, p. 12).

Constituye una paradoja que Uruguay siendo una excepción en el fútbol mundial, en el sentido que posee títulos a nivel de selección como a nivel de clubes, tenga tantos vacíos historiográficos. A su vez, llama la atención que el mérito de los logros del fútbol uruguayo haya salido de un núcleo reducido, debido a la escasez de nuestra población.

Pero constituye también una paradoja, que los vacíos historiográficos existieron hasta inicios de nuestro siglo si tenemos en cuenta el lugar que ocupa el fútbol en nuestra cultura y en nuestra identidad nacional. Existe un trabajo que puede considerarse icónico y relevante, aunque la mirada es más bien sociológica y antropológica, allí hay un gran esfuerzo para historiar el fútbol uruguayo. Se trata de la obra de Franklin Morales (1969), Fútbol: mito y realidad.

Tuvimos que esperar prácticamente cien años para que las ciencias sociales en Uruguay produzcan discursos explicativos e interpretativos en clave histórica. Lo que ocurrió en nuestro país, al igual que en la región, fue que los pocos trabajos sobre historia del deporte nacieron envueltos por la influencia de la Historia tradicional, positivista. A su vez, muchas de esas narrativas estaban en manos de periodistas que en su mayoría pertenecían a ámbitos fuera de los circuitos académicos. Estos trabajos, se parecen más a narrativas, semblanzas o ficción sentimental memorística antes que Historia del Deporte con rigor científico.

En respuesta a esto, autores como Juan Carlos Luzuriaga (2009) y Andrés Morales (2013) han producido obras historiográficas de referencia en nuestro país. El trabajo de Morales se constituye en uno de los pioneros en problematizar y realizar un abordaje teórico y metodológico del lugar que ocupa el fútbol en la identidad nacional del Uruguay. El autor intentó dar respuesta a la relación existente entre el fútbol, la política y la construcción de identidad entre 1916 y 1930. Como afirma el propio Morales en su libro, "interesa estudiar el significado del Uruguay del Centenario en la construcción de la identidad nacional y el lugar que tuvo el fútbol en el fuerte proceso de invención y resignificación de tradiciones que se dio en el período estudiado" (2013, p. 11). 
Dossiê | La modernización en el fútbol uruguayo: tensiones surgidas en torno al deterioro de los clubes y al profesionalismo en la segunda mitad del siglo XX (LABORIDO, Gastón)

Otro de los obstáculos que tuvo la Historia del Deporte en el Uruguay, fue que en el propio ámbito académico hasta las últimas décadas del siglo XX, se la consideraba sin valor económico y como algo vulgar. Desde ese punto de vista, concebían al deporte como un objeto de estudio incapaz de mostrar las más tenues representaciones de las relaciones sociales (Falco Genovez, 1998).

Si las pretensiones están en contribuir al campo profesional de la investigación histórica y aproximarnos al deporte realizando un abordaje desde el oficio de la Historia, necesariamente hay que caracterizarlo como objeto de estudio, para poder teorizar e interpretarlo en clave histórica.

La concepción positivista y dominante durante el siglo XX, concibe al deporte como natural, eterno, positivo, apolítico, transparente, igualitario y fraterno (Altuve, 2016). Si bien es cierto que la humanidad siempre realizó ejercicio físico con diferentes finalidades (lúdicas, competitivas, militares, religiosas); no podemos considerar deporte a aquellas actividades previas al siglo XIX, ya que se trataba de juegos y competiciones rituales cuya función social era bien distinta en cada una de esas sociedades, y bien diferentes a las que corresponde al deporte moderno y de nuestra época. En general, existe un consenso entre los sociólogos e historiadores en cuanto a esto.

Como señala R. Velázquez Buendía (2001), lo que hoy se conoce como deporte surgió mediante un proceso de transformación de juegos y pasatiempos tradicionales iniciado por las elites sociales y en el que tuvieron un papel clave las "publics schools" y los "clubs" ingleses. Por lo tanto, el deporte moderno como institución y fenómeno de las actuales sociedades es producto de una ruptura histórica, nació en Inglaterra, cuna de la Revolución Industrial y espacio clásico del modo de producción capitalista, a fines del siglo XVIII y en los albores del XIX, y adquiere una enorme complejidad social y cultural a partir de la segunda mitad del siglo XIX. La revolución industrial estableció la diferencia entre tiempo de trabajo y tiempo de ocio, en cual el deporte pasó a ocupar un lugar significativo.

De acuerdo a Manuel García Ferrando:

El deporte, fenómeno característico y destacado de las actuales sociedades de masas, esconde detrás de su aparente simplicidad una enorme complejidad social y cultural. Su aparente simplicidad surge del hecho de que el lenguaje y el simbolismo deportivos, basados en el cuerpo humano en 
Dossiê | La modernización en el fútbol uruguayo: tensiones surgidas en torno al deterioro de los clubes y al profesionalismo en la segunda mitad del siglo XX (LABORIDO, Gastón)

movimiento a la búsqueda de resultados destacables, son asequibles a todas las personas con independencia de su nivel social y cultural, lo que justifica su universalidad. (1990, p. 27).

A su vez, el deporte como institución social propia de las sociedades industriales, tiene una compleja organización, cargado de instituciones, parámetros organizativos, multiplicidad de roles nítidos y sin significación religiosa. En consecuencia, el deporte moderno posee características diferenciales que se originan en las circunstancias sociales y ambientales creadas por el desarrollo de la civilización industrial durante el siglo XIX. Posteriormente, fábricas y deportes se exportaron a prácticamente todo el resto del mundo, creando una nueva etapa de la que el deporte es parte substancial de este fenómeno.

El movimiento deportivo iniciado en Inglaterra tuvo en Uruguay un caldo de cultivo como en pocos países del mundo. La práctica de los deportes modernos surgió naturalmente en la colectividad británica. El deporte llegó a Montevideo en el siglo XIX, cuando los ingleses lo introdujeron en el Río de la Plata y en otras partes del mundo, de la mano del ferrocarril, intercambios con la marinería y de la acción de los colegios ingleses. Como señala J. C. Luzuriaga (2009), su difusión en la sociedad uruguaya siguió la misma lógica que en Gran Bretaña y en otros países: pasando de las elites al resto de la población en forma de cascada.

El fútbol tuvo un rápido proceso de adaptación y popularización en el Uruguay. Esto lo convierte en una de las prácticas sociales de identificación colectiva más importantes del Uruguay, en tanto es un fenómeno que trasciende las expresiones características propias y se convierte en algo total (social, cultural, político y económico). En tal sentido, el fútbol genera manifestaciones en otros espacios sociales y configura un amplio número de fenómenos que se integran dentro del mundo de lo simbólico.

En esta dinámica incluyente del fútbol -de totalidad y globalidad- la sociedad se retrata y representa, pero también se cohesiona para dar sedimento al sentido nacional (Dávila, 2003). El fútbol es un sistema de relaciones y representaciones, que produce una integración simbólica de la población alrededor de los múltiples componentes que tiene, produce o atrae; sea a partir de la práctica deportiva como de las esferas que lo rodean directa o indirectamente. (CARRIÓN, 2006, p. 177). 
Dossiê | La modernización en el fútbol uruguayo: tensiones surgidas en torno al deterioro de los clubes y al profesionalismo en la segunda mitad del siglo XX (LABORIDO, Gastón)

Este deporte funciona como un fenómeno generador de hábitos, valores y sentimientos que conforman parte de la propia cultura y crea numerosos lazos identitarios. En el caso de la historia del Uruguay, el fútbol fue uno de los elementos catalizadores de la forja de la identidad nacional (MORALES, 2013). Los mecanismos básicos de construcción de identidades se producen en la vida social a través de la puesta en escena de rituales que permiten la afirmación simbólica de un yo o un nosotros frente a un ellos. En este sentido, lo simbólico juega un papel fundamental, puesto que crea una imagen del país en el propio interior y exterior, que tiene mucho de invención. La Historia fue importante para ello, ya que los países latinoamericanos surgieron como estados independientes sin tener una idea de nación o una identidad nacional.

Si tomamos aportes para la construcción del campo de la Historia de Deporte, en este caso brasileño, Patricia Falco Genovez (1998) demuestra la necesidad de una Historia de Deporte que sea concebida como objeto de estudio de la Historia Social o de la Historia Cultural. Desde esta perspectiva y en sociedades altamente "deportivizadas" como la uruguaya, debe ser interrogado el fenómeno.

En esta misma línea, Victor Andrade de Melo y Rafael Fortes (2010) plantean que el deporte debe ser encarado como un objeto de estudio como cualquier otro para la Historia y que además, contribuye para entender a la sociedad como un todo.

A questão é simples e óbvia, mas deve ser relembrada inclusive em função de que é clara a característica multiprofissional do campo de investigação da História do Esporte (na qual atuam "historiadores de formação", mas também oriundos de outras áreas). O que mais interessa no estudo histórico das práticas corporais é perceber que os objetos expressam/representam de forma multifacetada um conjunto de dimensões de um quadro de tensões sociais no tempo e no espaço. (MELO y FORTES, 2010, p. 25)

La Historia del Deporte es una herramienta para entender la sociedad, por lo tanto es necesario pensar al fenómeno deportivo en clave teórica y conceptual, propia de las ciencias sociales. Eduardo Archetti (1984) se propuso hacer una lectura de las sociedades de Argentina y Brasil a partir del mundo simbólico del fútbol, para ilustrar algunos de los aspectos centrales de sus culturas y del "ethos" nacional. De acuerdo a este autor, 
Dossiê | La modernización en el fútbol uruguayo: tensiones surgidas en torno al deterioro de los clubes y al profesionalismo en la segunda mitad del siglo XX (LABORIDO, Gastón)

a través del fútbol no sólo es posible encontrar un conjunto de símbolos que ayudan a pensar y categorizar relaciones sociales y a reproducirlas sino que también esto tiene consecuencias sobre la manera cómo los actores sienten, ven y perciben el mundo que los rodea. (ARCHETTI, 1984, p. 4).

Eduardo Archetti sentó las bases para hacer de los deportes un objeto de estudio de las ciencias sociales y toma la noción de "arena pública" para analizar el fútbol, mostrando como el deporte era un espacio privilegiado para indagar ciertos elementos que la sociedad y la cultura ponen en escena.

El deporte puede ser leído, en su multidimensioanalidad, como uno de los escenarios privilegiados para observar las representaciones que una sociedad hace de sí misma, para interpretar modelos de socialización y de las conductas humanas, comprender las razones que otorgan fuerza simbólica a su repertorio identificatorio.

En el caso uruguayo, tenemos una sociedad altamente "deportivizada", o más bien, "futbolizada", esto supone que cotidianamente todo es discutido en términos deportivos. El fútbol invade todos los espacios de la cotidianeidad, transformándose en uno de los principales productores de identidades, constituyendo el mayor ritual secular de masas, produciendo la mayor facturación de la industria cultural (Alabarces, 1998). Esto lo convierte en nuestra "arena pública" por excelencia.

\section{El impacto de la modernización en el fútbol en Uruguay: los inicios del estancamiento y el deterioro del club social}

El deporte uruguayo vivió en la primera mitad del siglo XX un período de bonanza, teniendo al fútbol como deporte estelar. El mundo conoció a Uruguay por el fútbol, gracias a las medallas de oro conquistadas en los Juegos Olímpicos de 1924 y 1928. Contribuyó también a ese reconocimiento la organización y coronación en el primer Mundial de 1930. La etapa inicial del siglo XX fue el período más importante y fermental de todo el desarrollo deportivo en la historia de nuestro país; que a nivel futbolístico se coronó con la conquista del Campeonato Mundial de Fútbol realizado en Brasil en 1950, el reconocido "Maracanazo".

El fenómeno deportivo como práctica social propia de las sociedades industriales, tiene una compleja organización, cargado de instituciones, parámetros 
Dossiê | La modernización en el fútbol uruguayo: tensiones surgidas en torno al deterioro de los clubes y al profesionalismo en la segunda mitad del siglo XX (LABORIDO, Gastón)

organizativos, multiplicidad de roles nítidos y sin significación religiosa. Una de las instituciones fundamentales del deporte moderno es el club.

la unidad básica de la actividad deportiva es el club, que abarca desde el modesto club deportivo local, con su sencillez organizativa y su reducido número de miembros, al multimillonario club de deporte espectáculo y profesionalizado, que al igual que las modernas sociedades anónimas, presenta una densa red de intereses de signo muy diverso, y en el que participan numerosas personas, desde los deportistas profesionales hasta los socios y los espectadores en general, pasando por los directivos y técnicos. (GARCÍA FERRANDO, 1990, p. 27).

Los clubes fueron muy importantes para el desarrollo y la expansión del deporte. Éstos surgieron con el formato de clubes barriales debido a las características geográficas y demográficas de nuestro país. Para estudiar los clubes de fútbol, es que insistimos con la noción de "arena pública", con el objetivo de comprender la sociedad uruguaya a través del fútbol. Analizar los clubes de barrio permite observar cuestiones simbólicas, ya que los clubes son instituciones donde la política se presenta tanto en la forma de naturalización y reproducción a un orden social dominante, como donde es posible plantear una política de transformación y crítica a lo dominante como la construcción de la alteridad y respeto a las diferencias.

El barrio y su entretejido implica un sistema de relaciones sociales especializadas, una dimensión histórica y procesos de identidad. Trabajar con unidades de distinguibilidad como clubes sociales "permitirá abordar la apropiación e interiorización del complejo simbólico-cultural que encierra la ciudad. Es decir, poder observar la conformación y transformación de subjetividades, procesos identitarios, vínculos vecinales / comunitarios, diálogos transgeneracionales e interculturales" (CÁNEVA y MENDOZA, 2007a, p. 160).

Cáneva y Mendoza (2007b) realizan un recorrido histórico de la conformación de los clubes sociales de La Plata en Argentina, teniendo en cuenta que constituyen lugares especiales en el entramado urbano, en este sentido, el club nace, crece y se transforma junto a la ciudad. Estos autores llegaron a la conclusión de que los clubes platenses representan el crecimiento y el desarrollo de cada uno de los barrios, la participación en 
Dossiê | La modernización en el fútbol uruguayo: tensiones surgidas en torno al deterioro de los clubes y al profesionalismo en la segunda mitad del siglo XX (LABORIDO, Gastón)

comunidad, el compromiso de trabajo en conjunto, la solidaridad entre los vecinos y la unión familiar para participar en la vida social.

Los autores reconocen tres periodos. Si bien la periodización fue hecha para el caso de Argentina, es posible aplicarla a nuestro país. El primer periodo, emergencia de los clubes de fútbol (1890-1930), inicia cuando el fútbol llegó a Montevideo hacia fines del siglo XIX, cuando los ingleses lo introdujeron en el Río de la Plata y en otras partes del mundo, de la mano del ferrocarril, intercambios con la marinería y de la acción de los colegios ingleses. En ese contexto, Uruguay asistía a la conformación del Estado y de la nacionalidad. Tal como ocurrió en Buenos Aires, el fútbol se popularizó rápidamente en Montevideo durante la primera década del siglo XX, con la fundación de una gran cantidades de clubes. En la primera década del siglo XX el fútbol se expandió por los barrios de Montevideo.

A comienzos del siglo XX el Uruguay era un país prospero y optimista con gran desarrollo deportivo. La matriz del deporte uruguayo está dada por la política Batllista (1903-1930), que a su vez terminó siendo catalizador del proceso de construcción de identidades que se desarrolló en el fútbol uruguayo de los años veinte y treinta en torno a la llamada "generación olímpica". El periodo también se caracterizó por tener un ente rector del deporte, la Comisión Nacional de Educación Física (creada en 1915), que dedicó su primer período a organizar cada rama deportiva, por eso los clubes fueron fundamentales en este período. En suma,

Es en todo este período cuando se consolidan las formas de conducción del deporte uruguayo, que ya venían gestándose desde principios de siglo cuando José Batlle y Ordóñez percibió la importancia del deporte como generador de nacionalidad y aglutinador de voluntades. Esas formas son las que han predominado hasta el comienzo del siglo XXI, modelo que si bien dio sus frutos en su momento, hoy se revela no adecuado a la necesidad del deporte de competencia. Se ha denominado también a esta modalidad como "folklórica". (GOMENSORO, 2015, p. 21)

El segundo periodo, lo denominan Esplendor de los clubes de fútbol (entre las décadas de 1930 y 1960), allí los clubes se consolidan como institución de la vida barrial y comunitaria. Coincide con la época de bonanza económica producida por los buenos precios de los productos exportables como consecuencia de la segunda guerra mundial 
Dossiê | La modernización en el fútbol uruguayo: tensiones surgidas en torno al deterioro de los clubes y al profesionalismo en la segunda mitad del siglo XX (LABORIDO, Gastón)

y durante parte de la década del cincuenta por la guerra de Corea, generaron una expansión económica en la industria y el empleo (mejorando los niveles de ingreso, logrando elevar el nivel de vida de la población).

Esta nueva sociedad una sociedad de masas fue el caldo de cultivo para la explosión del fenómeno asociacionista más específicamente de clubes de barrio deportivos y sociales siendo este tipo de institución la de mayor desarrollo y sacándole protagonismo a las instituciones de inmigrantes las cuales fueron perdiendo sus objetivos iniciales.

Las bases del éxito del deporte uruguayo estuvieron en ciertas "ventajas comparativas", que sintetiza de esta manera:

Un país educado, bien alimentado, de fácil comunicación, homogéneo culturalmente; Organización precoz del deporte; Multiplicidad de clubes, plazas de deportes y otras instalaciones adecuadas, con gran cantidad de dirigentes y voluntarios comprometidos; Elusión de las dos grandes guerras mundiales; Gran repercusión social del deporte, lo que determinó un importante apoyo al deporte desde el Estado y la sociedad civil. (GOMENSORO, 2015, p. 29).

Esas "ventajas comparativas" más la crisis europea y las guerras ayudaron a los uruguayos a competir en igualdad de condiciones en los Juegos Olímpicos y Mundiales de fútbol. Otros deportes también fueron importantes y se lograron importantes triunfos. Además de los muy buenos resultados en sucesivos campeonatos sudamericanos de fútbol, básquetbol, ciclismo y alguna medalla en los Juegos Olímpicos de Los Ángeles (1932) y Berlín (1936) demuestran que el deporte uruguayo iba en crecimiento en todo el período. Los éxitos continuaron con el Mundial de fútbol de 1950, las medallas olímpicas en basquetbol (1952 y 1956), el vice campeonato mundial en ciclismo (Atilio François).

De acuerdo a Gomensoro (2015) “para estos resultados influyó seguramente el desarrollo deportivo experimentado, el buen momento económico que vivía Uruguay y la debilidad de los países participantes en la II Guerra Mundial (1939 - 1945)" (p. 29). El autor también agrega que el modelo del deporte uruguayo dio sus frutos debido al auge que había tomado la organización deportiva cimentada en el funcionamiento de clubes pequeños, medianos y grandes en todo el país. Esos clubes eran "dirigidos por una gran 
Dossiê | La modernización en el fútbol uruguayo: tensiones surgidas en torno al deterioro de los clubes y al profesionalismo en la segunda mitad del siglo XX (LABORIDO, Gastón)

cantidad de dirigentes voluntarios, con emprendedoras federaciones que los congregaban, en un contexto internacional donde el profesionalismo y la especialización no habían aún llegado a la mayoría de los deportes" (GOMENSORO, 2015, p. 29).

Llegando al tercer momento de la periodicidad propuesta por Cáneva y Mendoza (2007b), nos encontramos con la etapa del Deterioro de los clubes de fútbol (correspondiente a la década del 60 en adelante). En este periodo el club social comenzó a transitar su etapa de decadencia y a largo plazo supuso la pérdida de lugares de privilegio para el fútbol uruguayo, debido a fuerzas y tensiones internas y externas. Esto es lo que nos interesa problematizar.

Visto el breve panorama anterior, estamos en condiciones de retomar las preguntas que fueron planteadas al inicio de este trabajo: ¿Cómo actuó el proceso modernizador en el fútbol uruguayo? ¿Cuál fue el impacto en nuestro país a partir de la década del sesenta? ¿Qué tensiones subyacen al proceso?

El exitoso modelo de la primera mitad del siglo XX, ya percibía signos de agotamiento en la década del 50. Ricardo Piñeyrúa (2008) intentó dar explicaciones y generar una hipótesis para responder acerca del agotamiento del modelo uruguayo. De acuerdo a su análisis, hubo dos momentos de cambio que relegaron a Uruguay y a países similares. En primer lugar, una vez finalizada la Segunda Guerra Mundial y sobre todo a partir de 1947 con el inicio de la Guerra Fría y el traslado del deporte a nuevos planos, los bandos socialistas y capitalistas, entendieron que los resultados obtenidos internacionalmente en el deporte eran elementos relevantes de la propaganda de esos regímenes. En segundo lugar, también influyó el desarrollo de los medios de comunicación, la globalización y la transformación del deporte de bandera política a entretenimiento masivo.

El contexto mundial está marcado por el comienzo de transformaciones profundas, dando paso al proceso de modernización del y en el fútbol. Se trata de un fenómeno relativamente nuevo, por lo tanto los debates surgidos a partir de esos cambios, también son nuevos. A su vez, los procesos de globalización han acentuado más esas transformaciones, cuando en los setenta y ochenta adquieren características nuevas con enormes tentáculos que tocan diversos aspectos. En términos de S. Villena (2003), las transformaciones continúan y asistimos a un escenario en el que se desplaza desde los patrones internacionales, dominantes hasta ahora, hacia formas que tienen 
Dossiê | La modernización en el fútbol uruguayo: tensiones surgidas en torno al deterioro de los clubes y al profesionalismo en la segunda mitad del siglo XX (LABORIDO, Gastón)

un carácter más bien transnacional. La consecuencia directa en América Latina, termina siendo el debilitamiento de la articulación entre fútbol y nacionalismo que tanto funcionó en la primera mitad del siglo XX.

Esto significa que, como ocurre en otros ámbitos, la globalización no debe entenderse principalmente como una mayor difusión de esta práctica deportiva-espectáculo ni como una creciente articulación entre organizaciones ancladas nacionalmente (procesos que podrían denominarse más apropiadamente como "internacionalización" antes que como "globalización"), sino principalmente como un proceso de transformación y, más puntualmente, de transnacionalización de sus patrones de organización $y$, articulado a ello, de sus funciones sociales y simbólicas (VILLENA, 2003, p. 260).

A grandes rasgos este proceso tiene como modelo el formato y la organización del fútbol europeo. Esto generó y genera que el fútbol transitara por una serie de cambios (en los patrones del juego, el consumo y la organización, difusión mediática, afición, entre otros) que están insertos en los procesos de mercantilización, hipermediatización y transnacionalización. Se trata de fenómeno que es global, que tiene que asegurar su contenido estético y lúdico para que atraiga la atención de los espectadores, genere íconos para consumir y no agote.

El desarrollo de los medios de comunicación y la transformación del deporte en uno de los principales agentes de entretenimiento no hizo más que acentuar las diferencias y los ejes se trasladaron de las naciones y sus sistemas económico - políticos, a las regiones y sus mercados.

Como señala S. Villena (2003), la globalización del fútbol no sólo implica la transnacionalización de su campo, sino que también del campo político (articulación que hizo posible el uso nacionalista del fútbol). Lo que regula esto, son las leyes de la economía del mercado global.

Frente a este fenómeno, Uruguay mantuvo su funcionamiento y organización anterior, basado en la matriz originaria batllista de corte amateur, desorganizado, no planificado, dependiendo de la improvisación. El profesor Arnaldo Gomensoro en el año 2014, en el contexto de un encuentro académico nombrado "Uruguay y los mundiales desde el sur" (organizado por el GREFU -Grupo de Estudios de Fútbol del Uruguay-), presentó una ponencia llamada "Maracaná, que me hiciste mal y que sin embargo te 
Dossiê | La modernización en el fútbol uruguayo: tensiones surgidas en torno al deterioro de los clubes y al profesionalismo en la segunda mitad del siglo XX (LABORIDO, Gastón)

quiero". El titulo es muy gráfico, ya que de acuerdo a su visión, quedó impregnada y desactualizada la improvisación ante la preparación. En el imaginario colectivo está la idea que esa es la clave para obtener logros.

La modernización fue dejando afuera a los países pequeños o en desarrollo, que no podían invertir en entrenamiento ni en instalaciones o equipos muy costosos. La infraestructura deportiva pasó a ser determinante y las viejas Plazas de Deportes no pudieron competir ni siquiera en la región. "Por este motivo, junto a la crisis económica, la Dictadura y el poco interés que se aplicó al deporte, los resultados internacionales empezaron a ser cada vez más discretos, perdiendo el lugar de privilegio de etapas anteriores" (GOMENSORO, 2015, p. 32).

Una vez terminados los conflictos internacionales, las importaciones mermaron en precio y cantidad, las divisan decrecieron sustancialmente, derivando en un desastroso deterioro de los términos de intercambio, las balanzas comercial y de pagos se volvieron negativas y los ritmos de crecimiento del PBI se desaceleraron. En efecto, Uruguay y Latinoamérica padecieron uno de los períodos históricos más turbulentos de su existencia, marcado por el enrojecimiento progresivo de las cifras de los indicadores socio-económicos y la desestabilización de la situación político-institucional.

Una de las principales preocupaciones del Uruguay de los años 60 era la política económica y sus resultados. El primer gobierno blanco asumió el 1 de Marzo de 1959 con un claro objetivo: modificar la política económica. Llegados al gobierno introducen un cambio en la línea de limitar la acción del Estado, bajo los lineamientos del Fondo Monetario Internacional, que si bien Uruguay lo integra desde el inicio en 1947, nunca había aceptado las directivas de política económica del organismo internacional, que explicaba la inflación como una consecuencia del exceso de demanda de los asalariados. A medida que se aplicó las tendencias librecambistas y aperturistas se hicieron sentir las consecuencias de forma negativa en la población, agudizando las tensiones sociales y la radicalización de algunos discursos políticos.

Las décadas de los 60 y 70, estuvieron marcados por la decadencia de la situación económica, en tanto los frigoríficos y otras fuentes laborales poco a poco cerraron. En 1958 los inversores estadounidenses que estaban en el país se retiraron definitivamente y los únicos que quedaron en pie fueron el Frigorífico Nacional y el Artigas, de lo que 
Dossiê | La modernización en el fútbol uruguayo: tensiones surgidas en torno al deterioro de los clubes y al profesionalismo en la segunda mitad del siglo XX (LABORIDO, Gastón)

eran los Establecimientos Frigoríficos del Cerro S.A. (EFCSA). Finalmente la industria frigorífica desapareció de la zona en 1970.

Esto tuvo un fuerte impacto en el modelo de los clubes en Uruguay, generando enormes tensiones. En esta etapa los clubes de barrio comenzaron su decadencia al estar transitando una nueva era caracterizada por la individualidad y el avance del mercado. El periodo coincide con la implementación de políticas liberales llevándolas al extremo y la instauración de gobiernos de facto en la región.

Estos cambios que se produjeron se enmarcan en procesos mayores, que dan cuenta del pasaje de una sociedad moderna nacional a una posmoderna transnacional. Estas formas se inscriben en procesos mayores como la globalización, la mundialización y la transnacionalización que provoca cambios en la forma de organización de los espacios públicos tradicionales, la vida ciudadana y los modos de habitar el barrio y la ciudad. De acuerdo a Cáneva y Mendoza,

Como producto de estas transformaciones se puede observar como los espacios públicos tradicionales son reemplazados cada vez más por espacios construidos según la lógica del mercado y controlados por entidades privadas. Estas transformaciones en los niveles de organización de la vida institucional y social de la nación conlleva la multiplicación de las referencias desde las cuales los sujetos constituyen su identidad, ya que el descentramiento no es sólo de la sociedad sino también de los individuos, que ahora viven una integración parcial y precaria de las múltiples dimensiones y adscripciones que los conforman. (2007b, p. 54).

Aquellos imaginarios sociales que tenían los clubes barriales de espacio colectivo, de lazo comunitario, han dejado de tener potencia instituyente y fue dando paso a un nuevo modelo de club, con otros significados y nuevas formas de organización. En síntesis y a modo de cierre tomando aportes de Sergio Villena:

El modelo de tipo "clubes", en el que estos constituían un espacio de socialidad de carácter local y sin objetivos de lucro, está siendo desplazado por la formación de empresas de tipo sociedades anónimas, muchas de ellas con capital transnacional. Las gratificaciones simbólica y el reforzamiento de vínculos comunitarios son relegados ante el objetivo de obtener ganancias, provocando así una pérdida de capital social acumulado por la sociedad civil (ver, para el caso argentino, Frydenberg, 2000 y Palomino, s.f.), así como una privatización del capital simbólico y económico que generaron los clubes. Por 
Dossiê | La modernización en el fútbol uruguayo: tensiones surgidas en torno al deterioro de los clubes y al profesionalismo en la segunda mitad del siglo XX (LABORIDO, Gastón)

otra parte, existe una creciente racionalización gerencial, asociada a la emergencia de una tecnocracia internacional, la cual implica la pérdida del control administrativo de los equipos por parte de los aficionados y socios, transformados éstos en accionistas. (VILLENA, 2003, p. 261).

\section{Conclusiones}

El estudio del fenómeno futbolístico trasciende nuestra sociedad y se circunscribe en un marco mundial de la globalización de un deporte que es consumido como espectáculo en todo el mundo. El trabajo que realizamos nos permite reflexionar sobre diversas cuestiones, que pasaremos a sistematizar a continuación.

Primeramente, en lo que refiere a la producción historiográfica del Deporte en el Uruguay, queda en evidencia que aún hay un largo camino por recorrer. Sin pretender hacer un balance del estado de la cuestión sobre el tema, ya que no fue el objetivo de nuestro trabajo, es evidente que los primeros trabajos con rigor teórico y metodológico son de inicios del siglo XXI. Si bien los trabajos que existen son muy valiosos, aún persisten vacíos historiográficos.

En cuanto a las potencialidades de la Historia del Deporte, es evidente que hoy es fundamental utilizarla como objeto para entender a la sociedad. Una mirada del fútbol uruguayo con una perspectiva histórica y crítica, nos posibilita leer los dramas de nuestra sociedad y sus tramas culturales. Esto convierte al fútbol en nuestra "arena pública" privilegiada, que puede ser usada como medio para reflexionar sobre la sociedad.

Por lo tanto, para abrir esta posibilidad de análisis es que podemos pensar al deporte como un objeto de la Historia Social o de la Historia Cultural. Queda claro aquí, que el campo existe $y$ es necesario que investigadores locales contribuyan a la consolidación del mismo.

Es en esta línea, que se tomó al deporte como un foco, más precisamente al fútbol, para tratar de interpretar que ocurrió en el Uruguay en el contexto del fenómeno de la modernización. A partir del análisis de la dimensión simbólica y su articulación con las esferas sociales y políticas, queda en evidencia que la década del sesenta trajo cambios internos y externos al fútbol. A pasos agigantados, cada vez más asistimos a un fenómeno que toca con sus tentáculos diferentes ámbitos. 
Dossiê | La modernización en el fútbol uruguayo: tensiones surgidas en torno al deterioro de los clubes y al profesionalismo en la segunda mitad del siglo XX (LABORIDO, Gastón)

Como analizamos en la segunda parte del trabajo, a partir de un breve recorrido histórico y del análisis de la categoría "clubes" y de los efectos de modernización del fútbol, vimos que la globalización transformó los espacios públicos tradicionales que fueron remplazados por espacios construidos según la lógica del mercado controlado por entidades privadas. Esto fue llevando al deterioro de la sociedad uruguaya y de los clubes de fútbol, caracterizadas por la individualidad y el avance del mercado. Aquellos imaginarios sociales que tenían los clubes barriales de espacio colectivo, de lazo comunitario, han dejado de tener potencia instituyente que se producen en los contextos actuales.

Para terminar, entiendo que es importante reivindicar la importancia que tiene estudiar el fútbol desde perspectivas académicas ya que se necesitan miradas que den cuenta acerca de esas prácticas culturales masivas y cotidianas. La intención no es marcar la agenda, pero hay temas que pueden ser tomados como objeto de estudio en próximos trabajos: los impactos en las funciones sociales de los deportes en tanto arena pública para la elaboración de identidades socioculturales.

\section{Bibliografía}

ALABARCES, Pablo. “¿De qué hablamos cuando hablamos de deporte?” In: Nueva Sociedad. n. 154, p. 74-86, 1998.

ALABARCES, Pablo. "Los estudios sobre deporte y sociedad: objetos, miradas, agendas". In: ALABARCES, Pablo (coord.). Peligro de gol. Estudios sobre deporte y sociedad en América Latina. Buenos Aires: CLACSO, 2000, p. 11-32.

ALTUVE MEJÍA, Eloy. "Sociología del deporte, poder y globalización. Tendencias de la sociología del deporte en los últimos 25 años". In: Espacio Abierto. Maracaibo: vol. 25, n. 4, p. 77-93, 2016.

ARCHETTI, Eduardo. Fútbol y ethos. Buenos Aires: Flacso, 1984.

BOURDIEU, Pierre. "Deporte y clase social". In: aavv. Materiales de sociología del deporte. Madrid: Ediciones de La Piqueta, 1993, p. 57-82.

CÁNEVA, Virginia y MENDOZA, Hernán. "Clubes sociales: espacios de reconstrucción y consolidación de identidades urbanas". in: Cuadernos de H. Ideas. Año 1, n. 1, p. 154-175, 2007a.

CÁNEVA, Virginia y MENDOZA, Hernán. "El club social nace, crece y se transforma junto a la ciudad". In: Clubes platenses al rescate de lo colectivo (tesis). FPyCS, UNLP., 2007b.

CARRIÓN, Fernando. "El fútbol como práctica de identificación colectiva". In: Área de candela. Fútbol y literatura. Vol. I, p. 177-181, 2006. 
Dossiê | La modernización en el fútbol uruguayo: tensiones surgidas en torno al deterioro de los clubes y al profesionalismo en la segunda mitad del siglo XX (LABORIDO, Gastón)

DEMASI, Carlos. "Prólogo: Junto a la línea de cal”. In: Cuadernos de Historia 14. A romper la red. Miradas sobre el fútbol, cultura y sociedad. Montevideo: Biblioteca Nacional. 2014, p. 912.

FALCO GENOVEZ, Patricia. "El desafío de Clío: el deporte como objeto de estudio de la historia". In: Lecturas: Educación Física y Deportes, Revista Digital. Buenos Aires: n. 9, 1998. Disponible en: http://www.efdeportes.com/efd9/clio1e.htm

GARCÍA FERRANDO, Manuel. Aspectos sociales del deporte. Madrid: Alianza, 1990.

GARRIGA ZUCAL, José y LEVORATTI, Alejo. "Lo múltiple y lo fragmentado. Pistas para los estudios sociales del deporte". In: MORA, Bruno (coord.). Deporte y Sociedad. Encontrando el Futuro de los Estudios Sociales y Culturales sobre Deporte. Montevideo: UdelaR, 2018, p. 327332.

GOMENSORO, Arnaldo. Historia del Deporte, la Recreación y la Educación Física en Uruguay. Crónicas y relatos. Montevideo: IUACJ, 2015.

LUZURIAGA, Juan Carlos. El football del novecientos. Orígenes y desarrollo del fútbol en el Uruguay (1875-1915). Montevideo: Santillana, 2009.

MELO, Victor A.; FORTES, Rafael. "História do esporte: panorama e perspectivas". In: Fronteiras, V. 12, n. 22, p. 11-35, 2010.

MORALES, Andrés. Fútbol, identidad y poder (1916-1930). Montevideo: Fin de Siglo, 2013.

MORALES, Franklin. Fútbol: mito y realidad. Montevideo: Nuestra Tierra, 1969.

PIÑEYRÚA, Ricardo. “Frustración inexplicable". Revista digital Vadenuevo, n. 1, 2008.

VELÁZQUEZ BUENDÍA, Roberto. "El deporte moderno. Consideraciones acerca de su génesis y de la evolución de su significado y funciones sociales". In: efdeportes.com Revista Digital [revista-e], n. 36, $2001 . \quad$ Disponible en: $<$ http://www.efdeportes.com/efd36/deporte.htm>

VILLENA, Sergio. "El fútbol y las identidades. Prólogo a los estudios latinoamericanos". In: ALABARCES, Pablo (coord.). Futbologías. Fútbol, identidad y violencia en América Latina. Buenos Aires: CLACSO, 2003, p. 21-35.

VILLENA, Sergio. "Gol-balización, identidades nacionales y fútbol”. In: ALABARCES, Pablo (coord.). Futbologías. Fútbol, identidad y violencia en América Latina. Buenos Aires: CLACSO, 2003, p. 257-271. 